\title{
Wahlleistungsvereinbarung und
} Honorararzt

Die honorarärztliche Tätigkeit von Radiologen in Krankenhäusern ist eine gängige Praxis, die für niedergelassene Radiologen und Krankenhäuser grundsätzlich vorteilhaft erscheint. Nachdem in der jüngeren Vergangenheit aufgrund sozialversicherungsrechtlicher Fragen neue Probleme im Bereich des Honorararzts entstanden waren, hatte sich der Bundesgerichtshof (BGH) mit der Einbindung eines Honorararzts in eine Wahlleistungsvereinbarung auseinanderzusetzen. In seiner Entscheidung vom 16.10.2014 (Az.: III ZR 85/14) hatte der BGH über $\S 17$ Abs. 3 Satz 1 des Krankenhausentgeltgesetzes (KHEntgG) und die Frage zu entscheiden, ob durch diese Regelung der Kreis der liquidationsberechtigten Wahlärzte abschließend festgelegt ist oder nicht. In der gleichen Sache befasste sich schließlich das Bundesverfassungsgericht (BVerfG) in seinem Beschluss vom 03.03.2015 (Az.: 1 BvR 3226/14) mit weiteren wichtigen Detailfragen aus der Entscheidung des BGH.

\section{Sachverhalt \\ $\nabla$}

Eine private Krankenversicherung machte gegenüber dem beklagten Arzt (Facharzt für Neurochirurgie) aus seitens des Versicherten abgetretenem Recht einen Honorarrückzahlungsanspruch geltend. Der Facharzt für Neurochirurgie war in einer Gemeinschaftspraxis als Arzt niedergelassen und hatte mit einem privaten Krankenhaus eine Kooperationsvereinbarung abgeschlossen. Das Krankenhaus hatte den Facharzt für Neurochirurgie nicht angestellt. Eine Patientin aus der Praxis des Neurochirurgen unterzeichnete im Vorfeld einer geplanten stationären Wirbelsäulenoperation eine Vereinbarung über die Behandlung gegen Privatabrechnung mit dem Neurochirurgen. Mit dem Krankenhaus vereinbarte sie wenige Tage später einen Behandlungsvertrag über die stationären Leistungen und zugleich eine Wahlleistungsvereinbarung, in der der Neurochirurg nicht namentlich als Wahlarzt oder „gewünschter“ Stellvertreter eines Wahlarzts genannt war.

\section{Begriff des Honorararzts \\ $\nabla$}

Der BGH vertrat in seiner Entscheidung vom 16.10.2014 die Auffassung, dass unter einem Honorararzt ein Facharzt zu verstehen sei, derim stationären und / oder ambulanten Bereich des Krankenhauses ärztliche Leistungen für den Krankenhausträger erbringt, ohne bei diesem angestellt oder als Belegarzt oder Konsiliararzt tätig zu sein. Ein Honorararzt werde zeitlich befristet auf Honorarbasis tätig, wobei das Honorar mit dem Krankenhausträger frei und unabhängig von den Vorgaben der Gebührenordnung für Ärzte vereinbart werden könne und mangels Anstellung des Honorararzts keine tarifvertraglichen Bindungen bestünden.

Nach den Vorschriften des Krankenhausentgeltgesetzes (KHEntgG) werden vollstationäre und teilstationäre Leistungen der Krankenhäuser nach dem KHEntgG und dem Krankenhausfinanzierungsgesetz (KHG) vergütet ( $§ 1$ Abs. 1 KHEntgG). Unter den Oberbegriff der Krankenhausleistungen fallen dabei allgemeine Krankenhausleistungen und Wahlleistungen (§ 2 Abs. 1 Satz 1 zweiter Halbsatz KHEntgG). Detailregelungen zu den Wahlleistungen enthält $§ 17$ KHEntgG. Danach kann ein Patient unter den Voraussetzungen des $\S 17$ Abs. 1 bis 3 KHEntgG eine Vereinbarung über die Inanspruchnahme wahlärztlicher Leistungen mit dem Krankenhausträger treffen und auf diese Weise gegen Zahlung eines zusätzlichen Honorars sicherstellen, dass ihm die persönliche Zuwendung und besondere Qualifikation und Erfahrung des von ihm gewählten liquidationsberechtigten Arzts zuteil wird und zwar ohne Rücksicht darauf, ob er nach Art und Schwere der Erkrankung auf die Behandlung durch einen besonders qualifizierten Arzt angewiesen ist (BGH, Urteil vom 19.02.1998, Az.: III ZR 169/97). Der Kreis der in Betracht kommenden Wahlärzte wird durch $\S 17$ Abs. 3 Satz 1 KHEntgG festgelegt. Hiernach erstreckt sich eine Vereinbarung über wahlärztliche Leistungen auf alle an der Behandlung des Patienten beteiligten angestellten oder beamteten Ärzte des Krankenhauses, soweit diese zur gesonderten Berechnung ihrer Leistungen im Rahmen der vollstationären und teilstationären

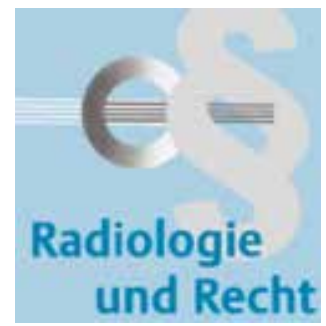

Behandlung berechtigt sind, einschließlich der von diesen Ärzten veranlassten Leistungen von Ärzten und ärztlich geleiteten Einrichtungen außerhalb des Krankenhauses (sog. Wahlarzt- oder Liquidationskette). Von der in $\S 17$ Abs. 1 Satz 1 KHEntgG normierten Wahlarztkette werden somit nicht alle an der Behandlung beteiligten Ärzte, sondern nur bestimmte Ärzte umfasst.

Nach dem eindeutigen Wortlaut des $§ 17$ Abs. 3 Satz 1 KHEntgG erstreckt sich, nach Ansicht des BGH, eine Wahlleistungsvereinbarung, deren wirksamer Abschluss Grundlage für die Abrechnung wahlärztliche Leistungen ist, auf angestellte oder beamtete Krankenhausärzte, denen der Krankenhausträger das Liquidationsrecht eingeräumt hat. Darüber hinaus erstrecke sich eine Wahlleistungsvereinbarung gemäß $§ 17$ Abs. 1 Satz 1 letzter Halbsatz KHEntgG auch auf die Leistungen von Ärzten und ärztlich geleiteten Einrichtungen außerhalb des Krankenhauses, soweit diese Leistungen im Rahmen der Behandlung des Patienten von angestellten oder beamteten Krankenhausärzten mit eigenem Liquidationsrecht veranlasst werden. Auf den Neurochirurgen traf diese externe Tätigkeit nicht zu, weil die Operation durch ihn nicht außerhalb des Krankenhauses erfolgte, sondern die vom Krankenhausträger geschuldete Hauptbehandlungsleistung war, die von dem Neurochirurgen aufgrund des Kooperationsvertrags gegenüber dem Krankenhaus erbracht wurde. Der BGH stellte sodann fest, dass der Kreis der liquidationsberechtigten Wahlärzte nicht durch $\S 17$ Abs. 1 Satz 2 KHEntgG erweitert werde. Diese Regelung betreffe die Einbringung diagnostischer und therapeutischer Leistungen als Wahlleistungen durch einen Arzt, ohne dass dieser beim Krankenhaus angestellt oder verbeamtet sein müsse. § 17 Abs. 1 Satz 2 KHEntgG befasse sich jedoch ersichtlich nur mit sogenannten medizinischen Wahlleistungen, z.B. der Anwendung einer bestimmten Methode oder dem Einsatz eines bestimmten Produkts und nicht mit der Person des Leistenden. 
Nichtigkeit der Vereinbarung über Behandlung gegen Privatrechnung

$\nabla$

Der BGH betonte daneben, dass aufgrund der abschließenden Festlegung des Kreises der liquidationsberechtigten Wahlärzte nach $\S 17$ Abs. 3 Satz 1 KHEntgG eine dem Schutz des Privatpatienten dienende zwingende preisrechtliche Norm einer externen Wahlleistungsvereinbarung entgegenstünde. Die Folge des Verstoßes gegen die zwingende preisrechtliche Norm ist die Nichtigkeit der externen Wahlleistungsvereinbarung, die der Neurochirurg mit dem Patienten abgeschlossen hatte.

Anders lag der Fall in einer früheren Entscheidung des BGH (Urteil vom 04.11. 2010, Az.: III ZR 323/09). Diese Entscheidung betraf den Anspruch eines externen Arzts gegen einen Wahlleistungspatienten auf Ersatz von Auslagen für aufgewendete Sachkosten. Der Patient hatte die private, persönliche Beratung und Behandlung durch die liquidationsberechtigten Wahlärzte des Krankenhauses vereinbart. Auf deren Veranlassung wurde in einer radiologischen Gemeinschaftspraxis eine Angiografie mit anschließender Dilatation der Arterien vorgenommen. Der BGH ging in diesem Fall davon aus, dass die Radiologen aufgrund der Vereinbarung wahlärztliche Leistungen nach Maßgabe des § 17 Abs. 3 Satz 1 KHEntgG auf Veranlassung des liquidationsberechtigten Arzts des Krankenhauses, das dem Patienten zur stationären Behandlung aufgenommen hatte, tätig geworden seien.

\section{Klarstellungen durch das Bundes- verfassungsgericht \\ $\nabla$}

Der vor den Zivilgerichten unterlegene Neurochirurg erhob gegen die Entscheidung des BGH Verfassungsbeschwerde. Diese nahm zwar das BVerfG nicht zur Entscheidung an, führte dessen ungeachtet in dem ablehnenden Beschluss (vom 03.03.2015, Az.: 1 BvR 3226/14) aus, dass Krankenhausleistungen nach $\S 2$ Abs.1 Satz 1 KHEntgG insbesondere ärztliche Behandlung, Krankenpflege, Versorgung mit Arznei-, Heil- und Hilfsmittel seien, die für die Versorgung im Krankenhaus notwendig seien, sowie Unterkunft und Verpflegung; sie umfassten allgemeine Krankenhausleistungen und Wahlleistungen (vgl. BVerfG, Beschluss vom 03.03.2015, Az.: 1 BvR 3226/14). Allge- meine Krankenhausleistungen seien nach der Definition des $\S 2$ Abs. 2 Satz 1 KHEntgG Krankenhausleistungen, die unter Berücksichtigung der Leistungsfähigkeit des Krankenhauses im Einzelfall nach Art und Schwere der Krankheit für die medizinisch zweckmäßige und ausreichende Versorgung des Patienten notwendig seien. Wahlleistungen seien nach $\S 17$ Abs. 1 Satz 1 KHEntgG ,andere als die allgemeinen Krankenhausleistungen“. Wahlleistungen dürften neben den Entgelten für die voll- und teilstationäre Behandlung gesondert berechnet werden, wenn die allgemeinen Krankenhausleistungen durch die Wahlleistungen nicht beeinträchtigt werden und die gesonderte Berechnung mit dem Krankenhaus vereinbart sei, § 17 Abs. 1 Satz 1 KHEntgG.

Das BVerfG führt in seiner Entscheidung aus, dass sich aus $\S 2$ Abs. 1 Satz 1 zweiter Halbsatz KHEntgG ergebe, dass neben den allgemeinen Krankenhausleistungen auch Wahlleistungen zu den Leistungen des Krankenhauses gehörten. Leistungserbringer der Wahlleistungen sei demnach das Krankenhaus, nicht der ausführende Arzt. Das Gesetz räume dem vom Krankenhaus insoweit berechtigten Wahlarzt in $\S 17$ Abs. 3 KHEntgG lediglich ein Liquidationsrecht für die von ihm durchgeführten wahlärztlichen Leistungen unmittelbar gegenüber dem Patienten ein. An dieser rechtlichen Leistungsbeziehung ändere sich durch das Tätigwerden eines Honorararzts nichts. Ein Honorararzt erbringe aufgrund eines Dienstvertrags im stationären oder ambulanten Bereich des Krankenhauses ärztliche Leistungen für den Krankenhausträger, ohne bei diesem angestellt oder als Belegarzt oder Konsiliararzt tätig zu sein. Die Tätigkeit des Honorararzts zeichne sich gerade dadurch aus, dass die rechtliche Grundlage seiner Leistungen nicht unmittelbar im Verhältnis zum Patienten bestehe, sondern gegenüber seinem Auftraggeber, dem Krankenhausträger.

Obwohl sich die Verfassungsbeschwerde mit dieser Problematik nicht weiter befasste, führte das BVerfG weiter aus, dass die wahlärztlichen Leistungen keine Leistungen des ausführenden Arzts, sondern des Krankenhausträgers seien. Für diese Leistung erhalte der Honorararzt eine Honorierung vom Krankenhausträger, deren Höhe das Ergebnis freier Vertragsverhandlungen sei. Die Honorarvereinbarung zwischen Honorararzt und Krankenhausträger sei nach der Rechtsauffassung des BGH, bestätigt durch das BVerfG, frei und unabhängig von den Vorgaben der Gebührenordnung für Ärzte oder etwaiger Tarifbindungen des Krankenhauses (vgl. BVerfG, Beschluss vom 19.03.2004, Az.: 1 BvR 1319/02). Der Neurochirurg wäre daher nicht gezwungen gewesen, die ärztlichen Leistungen zu erbringen, wenn er der Auffassung sei, ein zu geringes Honorar zu erzielen. Dem BVerfG fehlte es an weiteren Erläuterungen, warum dem Honorararzt dennoch ein Liquidationsrecht für eine Leistung verfassungsrechtlich garantiert sein solle, die bereits sein Auftraggeber, hier also der Krankenhausträger, gegenüber dessen Vertragspartner, dem Patienten, unter Heranziehung des Honorararzts als Auftragnehmer zu erbringen habe.

Das BVerfG stellt andererseits klar, dass die Entscheidung des BGH nicht auf der Annahme beruhe, ein Honorararzt könne (generell) keine wahlärztlichen Leistungen abrechnen. In dem konkreten Fall des Neurochirurgen war dieser weder als Wahlarzt noch als „gewünschter“ Stellvertreter eines Wahlarzts aufgeführt. Nach Auffassung des BVerfG hat sich der BGH daher nicht mit der Frage befasst, ob ein Honorararzt in der Wahlleistungsvereinbarung zwischen Krankenhausträger und Patienten als solcher bestimmt werde und in dieser Eigenschaft Leistungen abrechnen könne. Eine Aussage über den zulässigen Inhalt einer Wahlleistungsvereinbarung, insbesondere über die Zulässigkeit einer ausdrücklichen Bestimmung eines Honorararzts als Wahlarzt, sei in der Entscheidung des BGH nicht getroffen worden. Der BGH habe daher lediglich entschieden, dass der Honorararzt nicht in die Gruppe von Ärzten falle, die zwar nicht in der Wahlleistungsvereinbarung genannt werde, auf die sich die Vereinbarung aber nach $\S 17$ Abs. 3 Satz 1 KHEntgG „erstrecke“ und daneben, dass die Abrechnung wahlärztliche Leistungen nicht in Umgehung des $\S 17$ KHEntgG durch privatärztlichen Vertrag zwischen Honorararzt und Patienten vereinbart werden könne.

\section{Fazit}

$\nabla$

Das Thema „Honorararzt“ bleibt nach den Entscheidungen des BGH und des BVerfG, die den gleichen Fall betrafen, eine sensible Kooperationsform zwischen niedergelassenen Ärzten und Krankenhäusern. Sie wird durch die anstehenden Änderungen 
im Strafgesetzbuch und der Einführung der Straftatbestände der Bestechlichkeit und Bestechung im Gesundheitswesen ( $\S \S 299$ a und b StGB) neue Brisanz erhalten.

Soweit zunächst nach der Veröffentlichung der Entscheidung des BGH vom 16.10.2014(Az.: III ZR 85/14) der Eindruck entstanden war, dass honorarärztliche Tätigkeiten nicht Gegenstand einer Wahlleistungsvereinbarung sein können, hob das BVerfG in seinem Beschluss vom 03.03.2015 (Az:: 1 BvR 3226/14) hervor, dass die Entscheidung des BGH diese Rechtsfrage gerade nicht betreffe. Auch wenn in $\S 17$ Abs. 3 Satz 1 KHEntgG die angestellten Ärzte des Krankenhauses genannt werden, wollte der Gesetzgeber, wie es in der Begründung des Gesetzentwurfs des Fallpauschalengesetzes vom 11.09.2001 (BT-Druck 14/6893) sehr knapp heißt, die entsprechenden Vorschriften der Bundespflegesatzverordnung (BPflV) im Wesentlichen übernehmen. In § 22 Abs. 3 Satz 1 BPflV a.F. hieß es aber neutral: „Eine Vereinbarung über wahlärztliche Leistungen erstreckt sich auf alle an der Behandlung des Patienten beteiligten Ärzte des Krankenhauses“ eine Differenzierung nach angestellten und verbeamteten Krankenhausärzten erfolgte in $§ 22$ Abs. 3 Satz 1 BPflV nicht. Daher ist bei der Interpretation der Frage, ob „angestellter Arzt des Krankenhauses “ ein im Sinne von $\S 7$ SGB IV sozialversicherungsrechtliches Anstellungsverhältnis voraussetzt, die Begründung des Gesetzgebers heranzuziehen. Dieser führte die Sozialversicherungspflicht gerade nicht in seiner Gesetzesbegründung an, sondern wollte die frühere Vorschrift übernehmen.

Geklärt ist nach der Entscheidung des BGH vom 16.10.2014 (Az.: III ZR 85/14) nunmehr nur, dass der Honorararzt nicht in die Gruppe von Ärzten fällt, die zwar nicht in der Wahlleistungsvereinbarung genannt werden, auf die sich die Vereinbarung aber erstreckt. Für die Tätigkeit als Honorararzt folgt daraus, dass die Abrechnung von Leistungen eines Honorararzts nur dann als Wahlleistungen abgerechnet werden können, wenn der Honorararzt in der Wahlleistungsvereinbarung nament- lich als Wahlarzt oder als „gewünschter“ Stellvertreter eines Wahlarzts aufgeführt ist. Die (Wahl-)Leistungserbringung erfolgt aber stets durch den Honorararzt für den Krankenhausträger und nicht aus einem eigenen vertraglichen Rechtsverhältnis zwischen Patient und Honorararzt. Einer gesonderten Vereinbarung über die Privatabrechnung der Leistung seitens des Honorararzts attestiert der BGH die Nichtigkeit. Aus einer solchen Vereinbarung über Leistungen gegen Privatabrechnung kann daher der Honorararzt keinen Honoraranspruch gegen den Patienten ableiten.

\section{René T. Steinhäuser}

Rechtsanwalt

Rechtsanwälte Wigge

Neuer Wall 44

20354 Hamburg

Telefon: (040) 3398705-90

Telefax: (040) 3398705-99

Internet: www.ra-wigge.de

E-Mail: kanzlei@ra-wigge.de 